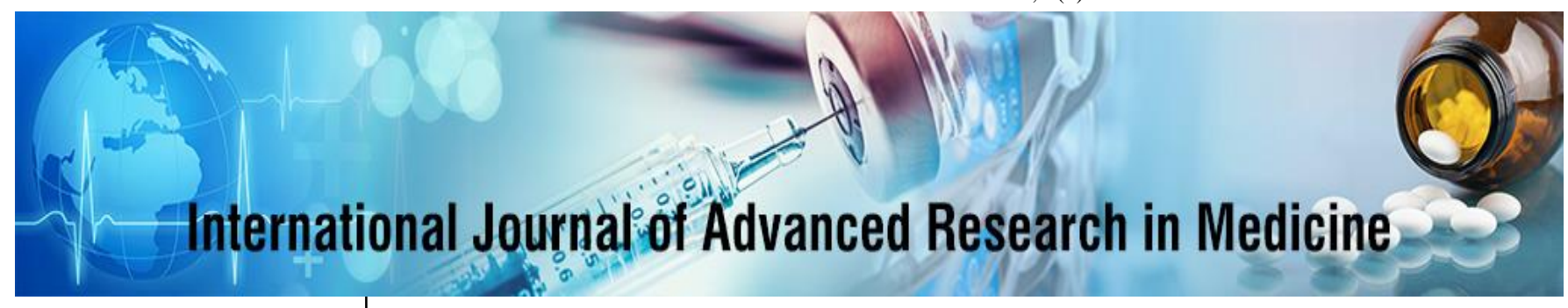

E-ISSN: 2706-9575

P-ISSN: 2706-9567

www.medicinepaper.net/ IJARM 2019; 1(2): 78-82 Received: 13-05-2019

Accepted: 15-06-2019

Athmar Jasim Mohammed Ministry of Health,

Baghdad, Iraq

Rafal Ismail Abbas Ministry of Health, Baghdad Al-Russafa Health Directorate, Madaen General Hospital, Baghdad, Iraq

Awatif Jaafar Sadeq Ministry of Health, Baghdad Medical Office, Al-Karkh, Karkh Maternity Hospital, Baghdad, Iraq
Corresponding Author: Athmar Jasim Mohammed Ministry of Health, Baghdad, Iraq

\section{Survey of diabetic pregnancies in Baghdad}

\author{
Athmar Jasim Mohammed, Rafal Ismail Abbas and Awatif Jaafar Sadeq
}

DOI: https://doi.org/10.22271/27069567.2019.v1.i2b.21

\begin{abstract}
A survey of some of the problems involved in pregnancy complicated by diabetes based on 34 years of experience from the Copenhagen center is presented.

The importance of centralization of the management is emphasized. The perinatal mortality among 1885 infants of diabetic mothers born in the period 1946 to 78 is analyzed; during the years a significant decrease from 22.1 to $4.4 \%$ is demonstrated. Congenital malformations are now counting for $50 \%$ of the perinatal death in diabetic pregnancies. Among infants whose mothers outside pregnancy attended two hospitals specialized in the treatment and ambulatory control of diabetes, the rate of major congenital malformations was significantly reduced compared to infants of mothers whose diabetes outside pregnancy were controlled elsewhere (5.6\% and $12.2 \%$ respectively).

The findings suggest that poor diabetic control outside pregnancy might be teratogenic.
\end{abstract}

Keywords: Emphasized, survey, diabetic pregnancies, ambulatory, teratogenic

\section{Introduction}

In 1989 the St Vincent declaration stated as a five-year goal that the "outcome of diabetic pregnancy should approximate that of the non-diabetic pregnancy ${ }^{[1]}$."

There are no prospective data from a large population base to determine whether this goal has been achieved. Within the former Northern region, data have been collected on perinatal mortality since 1982 and on fetal anomalies, since $1984^{[2,3,4]}$. In 1993, it was agreed to prospectively collect population-based information on the outcome of all diabetic pregnancies and to compare outcome with that of the background population.

The research presents the outcome data for all pregnancies in diabetic women booked in 1994.

This prospective nationwide study conducted during 1982-1985 examined the rates of hypertensive disorders, perinatal mortality and morbidity in 491 insulin-dependent diabetic pregnancies (White's classes: B, 164; C, 129; D, 172; F, 26) and in the total population of 279,000 . The rates of pregnancy-induced hypertension $(\mathrm{PIH})$ or pre-eclampsia $(20.6 \%)$, premature delivery $(24.6 \%)$, and cesarean section $(45.2 \%)$ in the diabetic group were more than four times higher than normal.

PIH or preeclampsia occurred significantly $(p<0.01)$ more frequently in patients with diabetic microangiopathy. Mean birth weight was similar in the two populations but gestational age was significantly $(p<0.001)$ shorter $(38$ weeks) in the diabetic group.

The rate of large for gestational age infants $(20 \%)$ in the diabetic group was considerably above normal $(3.5 \%)$. Although perinatal mortality rate in the diabetic group was only $3.1 \%$, it was 4.4 times higher than normal; five of ten fetal deaths were associated with poor glycemic control and thus may not really be unexplained.

Neonatal morbidity was significantly more frequent in the diabetic group; still, the incidence of idiopathic respiratory distress syndrome was only $1.6 \%$ compared with $0.6 \%$ in the general population. Discriminant analysis revealed that gestational age at birth and elevated maternal HbA1c values in early pregnancy independently of each other had a significant impact on the occurrence of neonatal morbidity.

A prospective study extending over 12 years has surveyed 107 latent diabetics and 172 clinical diabetics during pregnancy. Among the clinical diabetic's background, retinopathy was evident or developed during pregnancy in 40 cases. Eleven cases showed proliferative changes, only 1 such case appearing de novo during gestation.

There was a higher incidence of fetal loss in the diabetic than the latent diabetic group, and this loss was highest in those with proliferative retinal disease. Pregnancy was not associated with an increased risk to the mother of progression of retinal changes and visual loss. 
The only known direct relationship, namely, that between duration of diabetes and ocular complications, is confirmed. A major fear of both doctor and patient has been that pregnancy in the diabetic may precipitate or aggravate retinopathy and lead to blindness.

Because retrospective reports'-3 have been conflicting, a prospective survey was begun in 1967 and has involved 279 patients up to 1978 . The course of diabetic eye disease has been studied in relation to pregnancy, and eye ground signs have been evaluated, to see if they might in themselves be sufficient reason for termination of pregnancy. Findings from this study have been analyzed and are now presented. Patients and methods Patients attending the antenatal clinics at the Royal Women's Hospital, Melbourne, who had latent or clinical diabetes were seen on a regular basis throughout their pregnancies.

They were grouped into latent and clinical diabetics according to the recommendations of the World Health Organisation ${ }^{[4]}$. Latent diabetics had either abnormal glucose tolerance tests or frank hyperglycemia during pregnancy, the latter returning to normal after delivery. The clinical diabetics showed an abnormal response to the glucose tolerance test and symptoms or complications of diabetes. At each visit, both during pregnancy and after delivery, a full routine ocular examination was made with special note of any retinopathy.

Those Correspondence to Dr. Magda Horvat, 32 Gisborne St., East Melbourne, Victoria 3002, Australia. With overt retinopathy at their first visit were reexamined at intervals of 2 to 4 weeks, those with normal ocular fundi 4 to 8 weekly. Mothers were reviewed again in the puerperium and, where possible, 6 monthlies thereafter. 107 latent diabetics were followed up through 119 pregnancies and 172 clinical diabetics through 227 pregnancies. Over the last 3 years of the study, all new patients were submitted to routine stereo fluorescein angiography some 6 months after delivery.

Earlier patients who were still attending, and as many of the others who could be traced, were similarly examined-in all, a total of 93 patients. When the ophthalmoscopic and angiographic signs differed, angiographic pictures were taken as the basis for classification. It was made a general policy that this investigation was not performed during pregnancy. The retinal appearances in the clinical diabetics were classified at the patient's first presentation as normal, background, or proliferative. Included in background retinopathy were microaneurysms, varicose small vessels, retinal hemorrhages, retinal edema and 'hard' and 'soft' exudates. Stereo angiographic signs included one or more of the following features: capillary bed obliteration, capillary aneurysms, vessel wall varicosities, and staining. When new vessels were seen on the retina, on the disc, or in the vitreous, the retinopathy was labeled proliferative. The course of the retinopathy was classified under 3 headings: (1) No overall change. There was no observable change in clinical appearances or a

\section{Materials and Method}

Patients attending the antenatal clinics at the Royal Women's Hospital, Melbourne, who had latent or clinical diabetes were seen on a regular basis throughout their pregnancies. They were grouped into latent and clinical diabetics according to the recommendations of the World Health Organisation ${ }^{[4]}$. Latent diabetics had either abnormal glucose tolerance tests or frank hyperglycemia during Pregnancy, the latter returning to normal after delivery.

The clinical diabetics showed an abnormal response to the glucose tolerance test and symptoms or complications of diabetes. At each visit, both during pregnancy and after delivery, a full routine ocular examination was made with special note of any retinopathy.

Those Correspondence to Dr. Magda Horvat, 32 Gisborne St., East Melbourne, Victoria 3002, Australia. with overt retinopathy at their first visit were reexamined at intervals of 2 to 4 weeks, those with normal ocular fundi 4 to 8 weekly. Mothers were reviewed again in the puerperium and, where possible, 6 monthlies thereafter. 107 latent diabetics were followed up through 119 pregnancies and 172 clinical diabetics through 227 pregnancies. Over the last 3 years of the study, all new patients were submitted to routine stereo fluorescein angiography some 6 months after delivery. Earlier patients who were still attending, and as many of the others who could be traced, were similarly examined-in all, a total of 93 patients.

When the ophthalmoscopic and angiographic signs differed, angiographic pictures were taken as the basis for classification. It was made a general policy that this investigation was not performed during pregnancy.

The retinal appearances in the clinical diabetics were classified at the patient's first presentation as normal, background, or proliferative. Included in background retinopathy were microaneurysms, varicose small vessels, retinal hemorrhages, retinal edema and 'hard' and 'soft' exudates. Stereo angiographic signs included one or more of the following features: capillary bed obliteration, capillary aneurysms, vessel wall varicosities, and staining. When new vessels were seen on the retina, on the disc, or in the vitreous, the retinopathy was labeled proliferative. The course of the retinopathy was classified under 3 headings: (1)

No overall change. There was no observable change in clinical appearances.

All hospitals caring for diabetic pregnancy within the former Northern region participated. A steering group of diabetologists, obstetricians, and a neonatologist was formed and data collection from medical, obstetric, and neonatal notes agreed. Data were collected by an audit coordinator, who was notified when a woman with insulindependent or non-insulin dependent diabetes mellitus booked at an antenatal clinic.

The audit began in September 1993 and all sites were enrolled by December 1993. All diabetic pregnancies booked between 1 January and 31 December 1994 were notified. The last pregnancy notified proceeded to delivery in July 1995.

Data were analyzed by spas. Odds ratios for incidences and their $95 \%$ confidence intervals were calculated by standard methods [5]. Background population data for 1994 were obtained from the collaborative perinatal mortality survey 2 and the regional fetal abnormality survey ${ }^{[3]}$.

\section{Results}

There were 113 booked pregnancies in 111 diabetic women (two women booked with two pregnancies in the same year); 105 women were treated with insulin before pregnancy. Figure 1 shows the glycated hemoglobin concentration (hemoglobin A1c) plotted against the reference range for each hospital at booking (0-10 weeks' 
gestation) and in the second trimester (14-27+6 weeks). Several different assays for glycated hemoglobin were used by the hospitals (assay ranges were variable from $2.5-4.4 \%$ to $4.5-6.5 \%$ ).

The perinatal mortality rate was $48 / 1000$ for diabetic pregnancies compared with $8.9 / 1000$ for the background population (odds ratio 5.38; $95 \%$ confidence interval 2.27 to $12.70)$ and the neonatal mortality rate was $59 / 1000$ compared with $3.9 / 1000(15.0 ; 6.77$ to 33.10$)$. Two late neonatal deaths were due to congenital heart defects. Six percent of all fetal losses (6/109 cases) were due to major malformations. The congenital malformation rate was $83 / 1000$ compared with $21.3 / 1000(3.76 ; 2.00$ to 7.06$)$ in the background population.

Eight hospitals changed assay (and reference range) during the period of data collection. Seventy-one of the 113 pregnancies were planned. Prepregnancy advice was given in $57(80 \%)$ planned pregnancies. Despite this, however, measurements of diabetic control at booking in 59 women planning pregnancy showed values in only 17 (29\%) cases to be within the reference range; moderate control was recorded in 29 (49\%) cases and poor control in $13(22 \%)$.

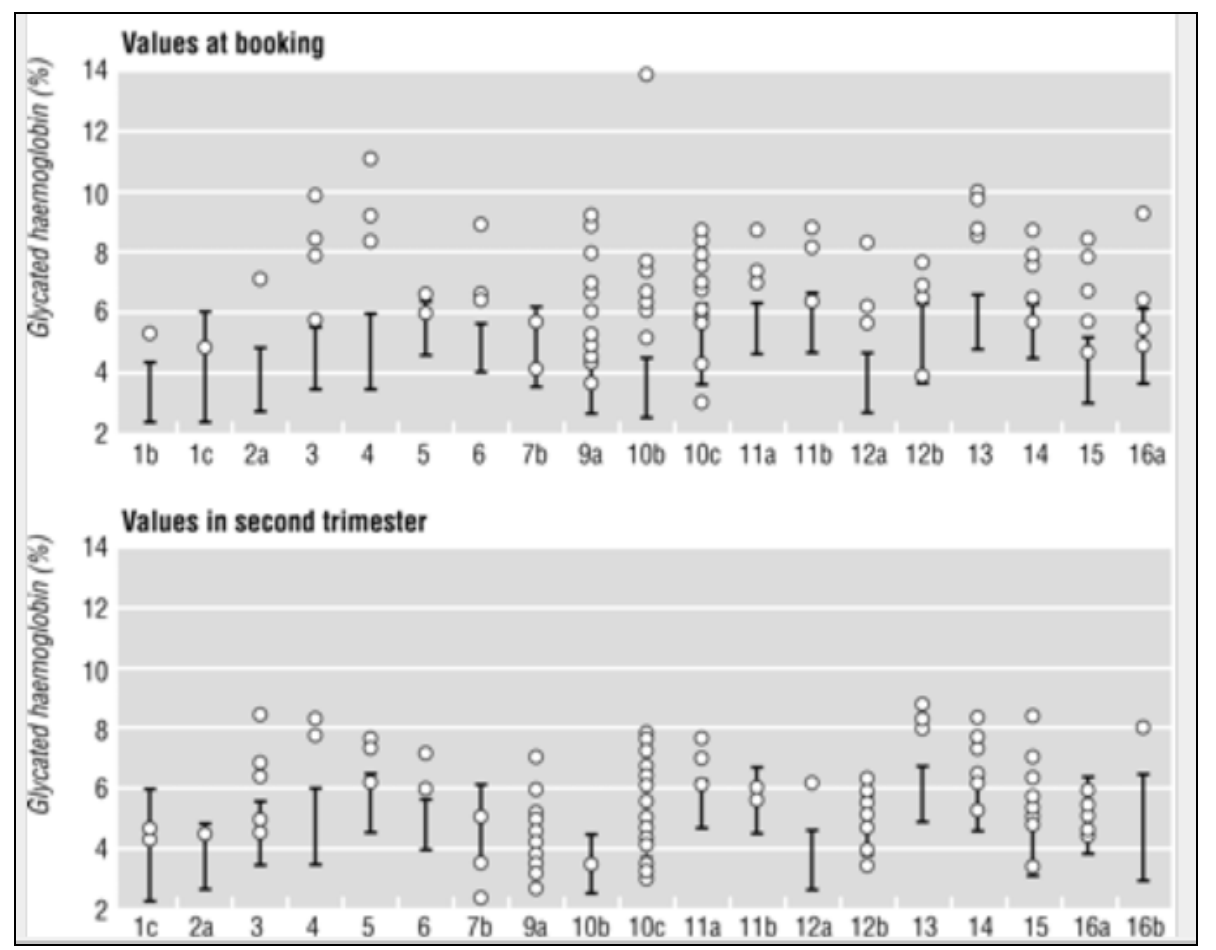

Fig 1: Glycated hemoglobin concentrations at booking and in second trimester. Bars are reference ranges for assay in each hospital. Points are values for each woman. Some hospitals used more than one assay (shown by a, b, c).

Thirty-eight women went into spontaneous labor, 19 before term. The cesarean section rate was $62 \%$ (59 cases) compared with background rates of $10.4 \%$ to $17.5 \%$ among the hospitals. Twenty-nine of the 113 pregnancies $(26 \%$; $95 \%$ confidence interval $17 \%$ to $34 \%$ ) had an adverse fetal outcome. Table 1 shows the absolute numbers of fetuses of diabetic women and their outcomes in relation to the background population. One hundred pregnancies lasted for at least 24 weeks. See table (1) below.

Table 1: Review attendance pattern-time related

\begin{tabular}{|c|c|c|c|c|}
\hline \multirow{2}{*}{ Follow-up } & Latent diabetics & Clinical diabetics & & \\
\cline { 2 - 5 } & & No retinopathy & Background & Proliferative \\
\hline Not after delivery & 52 & 44 & 8 & I \\
\hline 0-4 years after delivery & 55 & 43 & 20 & 3 \\
\hline 5-9 years after delivery & 0 & 27 & 9 & 3 \\
\hline 10+ years after delivery & 0 & 11 & 3 & 0 \\
\hline Total & 107 & 125 & 40 & 7 \\
\hline Mean follow-up period & 2 years & 5 years & 6 years & 4-5 years \\
\hline
\end{tabular}

Four were twin pregnancies, giving 104 fetuses. The perinatal mortality rate was 48/1000 compared with 8.9/1000 for non-diabetic births in 1994 (odds ratio 5.38; 2.27 to 12.70 ). The neonatal mortality rate was $59 / 1000$ live births compared with $3.9 / 1000$ (15.0; 6.77 to 33.10$)$.

A measure of global mortality is to account for all fetal losses from 20 weeks of gestation together with all postnatal deaths up to 1 year of age. The global loss rate was
$111 / 1000$ compared with a population average of $16 / 1000$ (6.9; 4.0 to 11.8$)$.

The congenital malformation rate was 9/109 fetuses (83/1000) compared with $21.3 / 1000$ alive at 20 weeks in $1994(3.76 ; 2.00$ to 7.06$)$. Confining the analysis to fetuses of over 24 weeks' gestation gave a rate of $5 / 104$ or $48 / 1000$ compared with a regional rate of $16.2 / 1000(2.99 ; 1.26$ to 7.05). 
Thirty-five percent of babies (36/104) weighed above the 95th centile for babies born in the Northern region ${ }^{[6]}$.

There was no systematic relation between the relative size of the babies and either death or serious congenital malformation. There were six live births and one stillbirth at less than 32 weeks of gestation. The odds ratio for live births at less than 32 weeks from diabetic pregnancies achieving viability was 6.2 (5.13 to 24.40$)$.

There was a substantial excess of premature births among diabetic pregnancies.

Table 2: Review attendance pattern — pregnancy-related

\begin{tabular}{|c|c|c|}
\hline & Latent diabetics & Clinical diabetics \\
\hline During 1 pregnancy but not after delivery & 42 & 39 \\
\hline During 1 pregnancy and after delivery & 54 & 80 \\
\hline Through 2 pregnancies and after delivery & 10 & 42 \\
\hline Through 3 or more pregnancies and after delivery & 1 & 11 \\
\hline Total patients & 107 & 172 \\
\hline
\end{tabular}

The clinical diabetics have not been subdivided because of the varia.

\section{Discussion}

We studied all pregnancies in diabetic women within a defined geographical region which was unbiased by differential referral or selective ascertainment. Global fetal and infant loss, perinatal mortality, neonatal mortality, and malformation rates were all significantly greater than those for the background population. Plainly the St Vincent declaration goal for pregnancy outcome was not being met in 1994.

The high fetal loss rate must be interpreted with caution, as the number of diabetic pregnancies remained small. However, a similar perinatal mortality rate and major malformation rate was reported a decade ago ${ }^{[7]}$.

Diabetic pregnancies under close scrutiny may yield a high detection rate of fetal anomaly. The major congenital malformation rate for diabetic pregnancy was similar to that in previous reports ${ }^{[8,9]}$. Some authorities maintain there is no further scope for improving the outcome of diabetic pregnancy ${ }^{[10]}$.

Though two-thirds of the pregnancies were planned by the mother, most women had not established good diabetic control before conception. Preconceptionally care reduces major congenital malformations 78 and the spontaneous abortion rate ${ }^{[11]}$. It is essential that we should improve delivery of this cost-effective care ${ }^{[12]}$.

A major difficulty was the lack of a standardized measure of diabetic control. Many different assays of glycated hemoglobin concentration are in use with different reference ranges. This is a serious problem for all audit in diabetes. For comparison, we defined good diabetic control as a measure of hemoglobin A1c concentration within the assay's reference range, moderate control as a hemoglobin A1c concentration up to $8.5 \%$, and poor control as a hemoglobin A1c concentration exceeding 8.5\% [13, 14].

The main finding of the Northern Diabetic Pregnancy Audit in 1994 was that one in four women with pregestational diabetes had a poor pregnancy outcome. Improvements in periconceptional care are recommended, though we recognizes that other, unknown factors are related to the increased fetal malformation rate.

\section{Conclusion}

Diabetic pregnancy remains a high-risk state with perinatal mortality and fetal malformation rates much higher than in the background population.

Messages. Though diabetic women who plan their pregnancies receive pre-pregnancy advice, most have poor diabetic control at conception In diabetic pregnancies the perinatal mortality rate is five times higher, the neonatal mortality rate 15 times higher, and the congenital malformation rate four times higher than in the background population

There is a substantial excess of premature births in diabetic pregnancies

The outcome of diabetic pregnancy remains poor; better uptake of preconceptionally care may improve outcome

Neonatal morbidity was assessed in the offspring of 878 mothers with gestational diabetes mellitus (GDM), 132 mothers with pre-GDM, and 380 control subjects. Compared with the control group, the GDM group had a higher incidence of complications, including macrosomia, hypoglycemia, hyperbilirubinemia, hypocalcemia, polycythemia, and major congenital anomalies $(P<0.05)$.

Although our GDM patients were stringently managed with diet or diet plus insulin, as indicated, and maintained almost euglycemic values, these neonatal complications could not be eliminated.

Our data may be consistent with observations published during the last decade that even subtle degrees of maternal hyperglycemia can have a detrimental effect on perinatal outcome.

Most neonatal complications readily respond to therapy if diagnosed and treated early and promptly. Macrosomia can have a detrimental effect on delivery (trauma) and later long-term implications during childhood. Tight metabolic control with diet and, when indicated, insulin treatment may be advantageous in reducing fetal birth weight. Criteria of how tight the metabolic control should be remaining to be accurately defined.

\section{References}

1. Northern Regional Health Authority. Collaborative surveys of perinatal, late neonatal and infant death in the Northern region 1983 to 1994. Newcastle upon Tyne: NRHA, 1995.

2. Northern Regional Health Authority. Regional fetal abnormality survey progress reports 19851994. Newcastle upon Tyne: NRHA, 1995.

3. Northern Regional Survey Steering Group. Fetal abnormality: an audit of its recognition and management. Arch Dis Child. 1992; 67:770-4.

4. Gardner MJ, Altman DG, Morris JA, Gardner MJ. Calculating confidence intervals for relative risks, odds ratios, and standardized ratios and rates. In: Gardner MJ, Altman DG, eds. Statistics with confidence. London: BMJ Publishing Group, 1989, 50-63. 
5. Tin W, Wadiyar UK, Hey EN. Selection biases invalidate current low birth weight weight-forgestation. Br J Obstet Gynecol (in press).

6. Traub AI, Harley JMG, Cooper TK, McGuinness S, Hadden DR. Is centralized hospital care necessary for all insulin-dependent pregnant diabetics? Br J Obstet Gynecol 1987; 94:957-62.

7. Steel JM, Johnstone FD, Hepburn DA, Smith AF. Can prepregnancy care of diabetic women reduce the risk of abnormal babies? BMJ. 1990; 301:1070-4.

8. Rosenn B, Miodovnik M, Combs CA, Khoury J, Siddiq i TA. Preconception management of insulin-dependent diabetes: improvement of pregnancy outcome. Obstet Gynecol. 1991; 77:846-9.

9. Gregory R, Scott AR, Mohajer M, Tattersall RB. Diabet ic pregnancy 1977-1990: have we reached a plateau? J R Coll Physicians Lond. 1992; 26:162-6.

10. Kitzmiller JL, Gavin LA, Gin GD, Jovanovic-Peterson L, Main EK, Zigrang WD. Preconception care of diabetes. Glycemic control prevents congenital anomalies. Diabetes Care. 1991; 265:731-6.

11. Elixhauser A, Gabbe SG, Weschler JM, Herman WH, Kitzmiller JL, Kaufman RC et al. Cost-benefit analysis of preconception care for women with established diabetes mellitus. Diabetes Care. 1993; 16:1146-57.

12. Diabetes Control and Complications Trial Research Group. The effect of intensive treatment of diabetes on the development and progression of long-term complications in insulin-dependent diabetes mellitus. $\mathrm{N}$ Engl J Med. 1993; 329:977-86.

13. Miller E, Hare JW, Cloherty JP, Dunn PJ, Gleason RE, Soeldner S et al. Elevated maternal hemoglobin A1c in early pregnancy and major congenital anomalies in infants of diabetic mothers. N Engl J Med. 1981; 304:1331-3. 